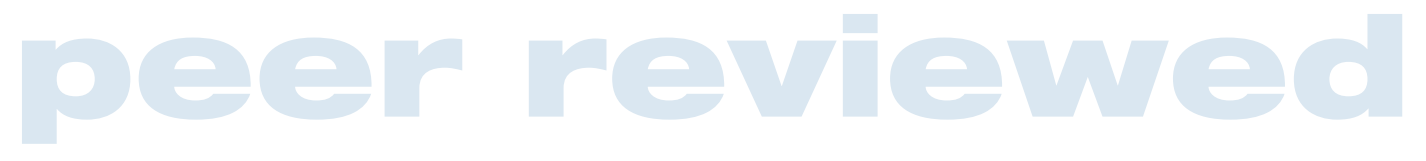

\title{
Perceptions of animal physiotherapy amongst Irish veterinary surgeons
}

\author{
Aoife Doyle and N. Frances Horgan \\ School of Physiotherapy, Royal College of Surgeons in Ireland, 123 St Stephen's Green, Dublin 2, Ireland
}

The aim of this study was to investigate veterinary surgeons' perceptions, knowledge and use of animal physiotherapy in the Republic of Ireland. A questionnaire was developed and sent to 200 veterinary surgeons, of which 97 were returned. Results indicated that 77 (79\%) of respondents were aware of animal physiotherapists. Common sources of information included veterinary colleagues, owners and professional journals, with physiotherapists themselves and undergraduate training being less commonly cited. Awareness of animal physiotherapy was greatest amongst those working in equine practice $\left(\chi^{2}=5.7, \mathrm{df} 1\right.$, $\left.p=0.017\right)$; they were more knowledgeable about its techniques ( $t=2.806, \mathrm{df} 75, \mathrm{p}=0.006)$ and more likely to refer $\left(\chi^{2}=48.36, d f 1, p=0.0001\right)$. Seventy-four respondents (96\%) thought that more research was necessary to increase the evidence base for animal physiotherapy. If this branch of physiotherapy is to develop, there needs to be increased interaction and co-operation between veterinary surgeons and chartered animal physiotherapists.

Irish Veterinary Journal

Volume 59 (2) 85-89, 2006

Keywords: Animal physiotherapy, Veterinary surgeons.

\section{Introduction}

Traditionally, physiotherapy has been applied to human clients in a hospital, community, industrial or private setting. However, a variety of physiotherapy techniques have been applied to animal patients including techniques of mobilisation/manipulation in the management of back and neck problems, heat and cold therapy, hydrotherapy, ultrasound and low level laser therapy, and exercise (Bromiley, 1991). A variety of conditions are seen by animal physiotherapists including: back and neck problems, muscular disorders, wounds, sprains/strains, and post-fracture rehabilitation (Hodges and Palmer, 1993; Knowles and Mackintosh, 1994; Marr et al., 1993; McNamara and Mackintosh, 1993).

There are published reports on the application of physiotherapy, with modifications, in animal patients (Bromiley, 1993; Hodges and Palmer, 1993; Knowles and Mackintosh, 1994; Mills and Levine, 1997; O'Connor, 1988; Porter, 1988). Herrod-Taylor (1967) reported successful outcomes of spinal manipulation in horses. Sharifi and Sharma (1990) reported earlier functional recovery in calves with tibial transfixation in reponse to exercise and massage. White and White (1995) described the physiotherapy management of a purebred Canadian Holstein female calf, who suffered from complete lack of tone in the muscles of both hind limbs, following assisted delivery.

\section{Author for Correspondence:}

Dr N Frances Horgan

School of Physiotherapy, Royal College of Surgeons in Ireland

123 St Stephen's Green, Dublin 2

Tel : +353 | 4022472

Fax : +353 | 402247 |

Email: fhorgan@rcsi.ie
The treatment involved a regime of massage (stretching and passive movements lasting 20 minutes were applied to each leg and repeated every eight hours) and aimed to overcome contracture of the extensor muscles of the hind limbs. The calf wore an adjustable splint and, after 10 days, the calf was able to stand. At 19 months the animal was able to walk normally.

Taylor (1970) described the treatment of trunk fibrositis in four elephants using short-wave diathermy; after a month of diathermy, the animals were able to feed themselves. Several authors reported the benefits of therapeutic ultrasound in the treatment of musculoskeletal problems in horses, dogs and cats (Antikatzides, 1986; Cramp, 1998; Lang, 1980; Morcos and Aswad, 1978). The application of alternative therapy, including acupuncture has been described (Bromiley, 1991). However, despite encouraging findings, some of the studies were limited by small sample sizes, lack of standardisation of treatments, subjective measures of outcome and the lack of control groups.

McNamara and Mackintosh (1993) surveyed veterinary surgeons in the UK and found that only $37 \%$ were aware of the existence of animal physiotherapists. Previous work in an Irish setting explored perceptions of animal physiotherapy among Irish undergraduate veterinary students (Ryan and Finn, 2000).

The aim of this study was to investigate veterinary surgeons' perceptions, knowledge and use of animal physiotherapy in the Republic of Ireland.

\section{Materials and methods}

A questionnaire was distributed to a random sample of 200 members on the Veterinary Register of Ireland, in October 2003, to ascertain their awareness and knowledge of animal physiotherapy. The total number of veterinary surgeons on the Veterinary Register of Ireland 
was 2,282 in 2003. The research instrument was developed following consultation with veterinary surgeons, and a review of relevant literature, to ensure face and content validity. The initial questionnaire was piloted and altered in response to veterinary surgeons' comments.

The questionnaire had three sections comprising of open-ended and closed-ended questions. The first section provided a demographic profile of the respondents, practice details and experience of referring animals to an animal physiotherapist. For the statistical analyses we assigned the respondents to two groups: veterinarians who qualified in the 1970s or earlier; and those who graduated in the 1980s or later. The second section explored the veterinary surgeons' awareness of the role of physiotherapy in the treatment of various physiotherapeutic techniques. The third section contained II statements, which examined veterinary surgeons' perceptions and attitudes towards animal physiotherapy. The respondents were asked to grade their responses using a Likert scale ranging from 'strongly agree' to 'strongly disagree'. Each questionnaire was accompanied by a cover letter, which explained the purpose of the study including details of how to return the completed questionnaire. Reminder letters were sent after a four-week period. Confidentiality of all responses was assured.

The study was approved by the Research Ethics Committee of the Royal College of Surgeons in Ireland. In addition, the Veterinary Council of Ireland gave permission to use the Veterinary Register of Ireland. The data were analysed using descriptive and inferential statistics using an SPSS package. Where appropriate, Chi-squared analysis, Pooled t test and a Mann Whitney- $U$ test were used to analyse data further. The level of significance was $p<0.05$.

\section{Results}

Of the 200 questionnaires distributed, 97 (49\%) were returned: 30 by veterinary surgeons in mixed practices; 21 by veterinarians in large animal practices, 18 from equine practices, 17 by those in small animal practices and II from education research and other areas of practice. The respondents had graduated from a variety of veterinary colleges; 77 from University College Dublin, nine from Trinity College Dublin and the remaining II from the Royal Veterinary College London, Berlin, Queensland, Edinburgh and Glasgow. Only eight of the respondents had graduated since 2000, 29 had qualified in the 1990s, 22 in the 1980s, 24 in the 1970s, 10 in the 1960s. Only four had been qualified for over 50 years

\section{Awareness}

The majority ( $n=77: 79 \%$ ) were aware of animal physiotherapists, while 35 (45\%) respondents were aware of the term 'chartered' animal physiotherapist. Twenty (21\%) of the veterinarians were not aware of these terms. Of those who were aware of animal physiotherapy $(n=77)$, the sources of information (more than one allowed) were veterinary colleagues ( $n=21: 27 \%)$, animal owners $(n=20: 26 \%)$, professional journals $(n=18: 23 \%)$ and physiotherapists $(n=13: 17 \%)$. Only four respondents (5\%) cited lectures at college, while eight (10\%) reported television and the Internet as sources.

Only $20(26 \%)$ of the veterinary surgeons who were aware of animal physiotherapists had referred a case to an animal physiotherapist. Of those 20, 12 indicated that they referred to a chartered physiotherapist who was a member of the Irish Society of Chartered Physiotherapists, two referred to unregistered physiotherapists, while six were unsure whether or not the physiotherapist was registered.

FIGURE I:Veterinary surgeons' awareness of use of physiotherapy for particular conditions $(n=77)$.

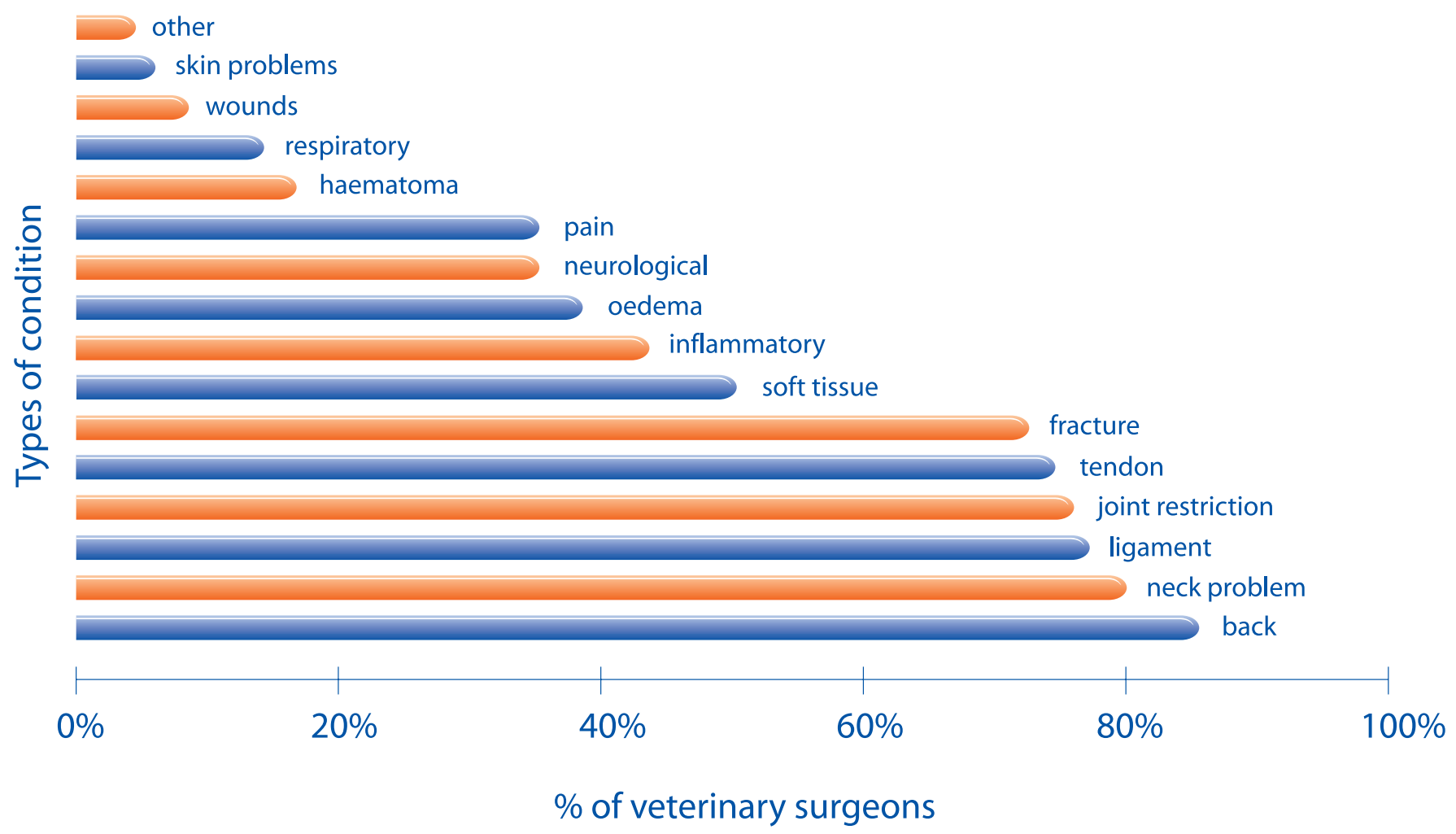


TABLE I: Self-assessment of knowledge of physiotherapeutic techniques by 77 veterinary respondents

\begin{tabular}{|c|c|c|c|c|c|}
\hline Technique & Know a lot & $\begin{array}{l}\text { Know much } \\
\text { about }\end{array}$ & $\begin{array}{l}\text { Know some- } \\
\text { thing about }\end{array}$ & Have heard of & Never heard \\
\hline \multicolumn{6}{|c|}{ Manipulative procedures } \\
\hline Manipulation & 5 & 17 & 47 & 29 & 2 \\
\hline Joint mobilisation & 4 & 10 & 43 & 26 & 17 \\
\hline Massage & 10 & 16 & 48 & 25 & 1 \\
\hline Taping & 0 & 8 & 14 & 16 & 62 \\
\hline Soft tissue friction & 1 & 5 & 22 & 25 & 47 \\
\hline \multicolumn{6}{|c|}{ Electrotherapy modalities } \\
\hline Ultrasound & 8 & 18 & 45 & 26 & 3 \\
\hline Laser & 6 & 14 & 33 & 33 & 14 \\
\hline Ultraviolet & 1 & 5 & 19 & 32 & 43 \\
\hline Ice therapy & 12 & 13 & 32 & 26 & 17 \\
\hline Short wave & 1 & 7 & 18 & 25 & 49 \\
\hline Interferential & 1 & 7 & 9 & 18 & 65 \\
\hline Infra-red & 3 & 13 & 26 & 31 & 27 \\
\hline \multicolumn{6}{|l|}{ Transcutaneous } \\
\hline Nerve stimulation & 2 & 13 & 22 & 38 & 25 \\
\hline Muscle stimulation & 2 & 12 & 18 & 43 & 25 \\
\hline \multicolumn{6}{|c|}{ Mobilisation procedures } \\
\hline Treadmill & 4 & 18 & 43 & 23 & 12 \\
\hline Passive exercises & 3 & 17 & 35 & 28 & 17 \\
\hline Active exercises & 4 & 18 & 38 & 26 & 14 \\
\hline Hydrotherapy & 10 & 23 & 38 & 20 & 9 \\
\hline Acupuncture & 5 & 14 & 47 & 29 & 5 \\
\hline
\end{tabular}

Thirteen of the veterinary surgeons who had referred an animal were satisfied with the treatment, four were very satisfied, three were unimpressed but not negative; none were dissatisfied. Those working in equine practice were more aware of animal physiotherapy $\left(\chi^{2}=5.7\right.$, df I, $\left.p=0.017\right)$ and more likely to refer to an animal physiotherapist $\left(\chi^{2}=48.36\right.$, df $\left.\mathrm{I}, \mathrm{p}=0.000 \mathrm{I}\right)$. There was no significant association between the year of qualification and level of awareness of animal physiotherapy $\left(\chi^{2}=0.3587, \mathrm{df} \mathrm{I}, \mathrm{p}=0.5492\right)$. When asked to nominate the most appropriate species to receive physiotherapy, 73 of the respondents opted for the horse, 69 for the dog, 24 for the cat and 13 for the cow; only nine respondents chose goats and exotic animals. Competitive animals (greyhounds, race horses, show jumpers) were considered most likely to receive treatment (59 respondents). There appeared to be greatest awareness of physiotherapy related to back (68 respondents: $88 \%$ ) and neck (62: $80 \%$ ) problems followed by ligament damage (61: 79\%), joint restriction (59:77\%), tendon damage (58: 75\%) and post-fracture rehabilitation (57: 74\%) (Figure I). Fewer veterinary surgeons were aware of physiotherapists' involvement with respiratory conditions (nine: 12\%), haematoma (11: 14\%) and skin problems (three: $4 \%$ ). The veterinary surgeons were asked to indicate their level of knowledge of a variety of therapeutic techniques (Table I). Techniques such as massage, ice therapy and hydrotherapy were well known, while respondents had a reasonable knowledge of manipulation / mobilisation. Electrotherapy treatments such as ultraviolet, shortwave diathermy and interferential were not known, while $62 \%$ had never heard of taping.

A knowledge score was generated by ranking the response from zero to four for each of the 19 techniques (i.e., $0=$ never heard of, $4=$ know a lot; maximum score $=76$ ). A pooled t test was performed to compare the mean knowledge between the referring veterinarians $(n=20$; mean score 34.0 , sd 10.7) and non-referring veterinary surgeons ( $n=57$; mean score 25.9 , sd 10.7). A significant difference was found $(t=-2.806, d f 75, p=0.006)$. A Mann Whitney $U$ test also indicated that referring veterinary surgeons had the greater knowledge of physiotherapy techniques $(Z=-2.54, p=0.01 \mathrm{I})$.

\section{Opinions}

The responses of the subset of veterinary surgeons aware of animal physiotherapy (77: 79\%), were explored in relation to II statements which referred to professional issues pertaining to animal physiotherapy (Table 2). Analysis indicated that 64 respondents (83\%) believed that the appropriate career path was through qualification as a chartered physiotherapist, followed by post-graduate qualification as an animal physiotherapist, and 7 I (92\%) agreed that a register of chartered animal physiotherapists should be set up in the Republic of Ireland.

A majority (74: $96 \%$ ) stated that more research needs to be published on the effect of animal physiotherapy and 69 (90\%) were interested in learning more about animal physiotherapy. While 70 (91\%) respondents indicated they would be willing to allow animal physiotherapists to choose their own treatments, under veterinary control, only 33 (43\%) believed that animal physiotherapists possess the ability to assess and evaluate musculoskeletal and neurological disorders. Less than half (35: $43 \%$ ) of the veterinary surgeons felt that animal physiotherapy was often used as a last resort and 53 respondents $(63 \%)$ believed the cost of animal physiotherapy did not 
TABLE 2: Responses of veterinary surgeons $(n=77)$ to II statements relating to animal physiotherapy

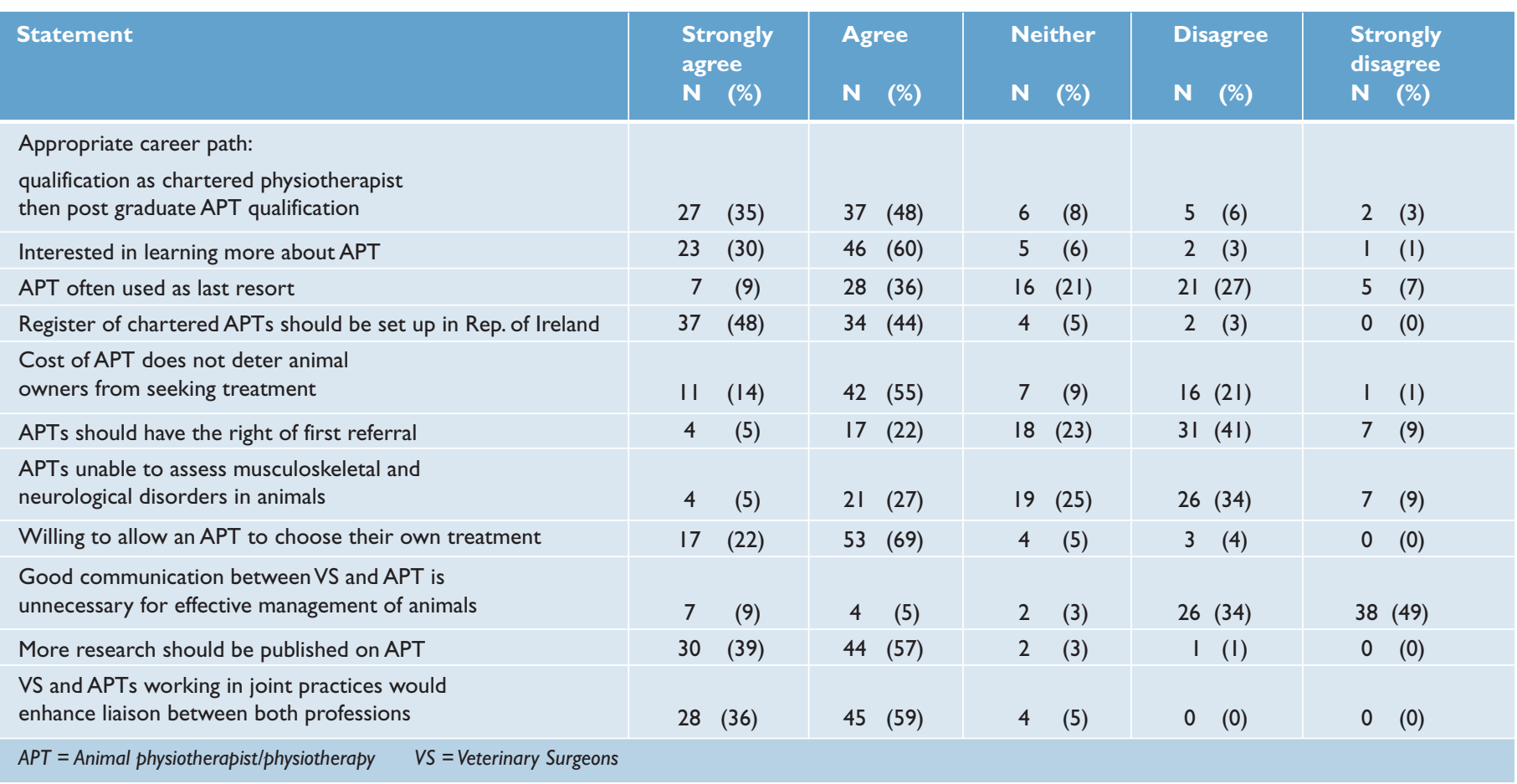

deter animals owners from seeking treatment.

Mann-Whitney $U$ tests were used to explore the differences in opinions between referring and non-referring veterinary surgeons for each statement. There were no significant differences between the groups ( $p>0.05)$. The opinions of the respondents were independent of whether or not they had referred to an animal physiotherapist.

In the final comments section the veterinary surgeons raised some interesting points. Generally, respondents felt that veterinary surgeons and the public should be given more information explaining the role of animal physiotherapy and its efficacy. Many were concerned that there was a large number of unqualified 'quacks' calling themselves animal physiotherapists treating animals. Several respondents indicated that they were very interested in referring to an animal physiotherapist but unable to locate one.

\section{Discussion}

The majority of respondents (79\%) were aware of animal physiotherapy. McNamara and Mackintosh (1993) reported that only $37 \%$ of UK veterinary surgeons were aware of the existence of animal physiotherapists. However, six years later, Shipley (1999) reported that $98 \%$ of veterinary practitioners in England and Wales were aware of them. Previous Irish work among fourth and fifth year undergraduate veterinary students at University College Dublin reported a level of awareness of $85 \%$ (Ryan and Finn, 2000). Veterinary colleagues, animal owners, professional journals and physiotherapists were the main sources of information about animal physiotherapy. Interestingly, only $8 \%$ of respondents cited undergraduate lectures at college.

The veterinary surgeons had considerable awareness of conditions appropriate for referral to physiotherapy. Many of these, such as back and neck injury or soft tissue and joint problems, are conditions for which physiotherapy has been publicised in human clients and it may be that their knowledge of human physiotherapy was extrapolated to the area of animal physiotherapy. Wounds and skin problems were considered least likely to benefit from physiotherapy, although Knowles and Mackintosh (1994) found that animal physiotherapists frequently treated wounds and skin problems. The results highlighted the need for more information on the less familiar conditions that may benefit from physiotherapy.

Consistent with the findings of Knowles and Mackintosh (1994), respondents were aware of physiotherapy techniques such as massage, hydrotherapy, ice therapy, manipulation/mobilisation and ultrasound. There has been much published literature relating to these techniques and their application in animals (Cramp, 1998; Lang, 1980; Morcos and Aswad, 1978).

The positive opinions of veterinary surgeons towards many aspects of animal physiotherapy were independent of whether or not they had ever referred to an animal physiotherapist. The referring veterinary surgeons had a significantly greater level of knowledge of the discipline. Perhaps this understanding of the role of the animal physiotherapist was involved in their decision to refer, or they may have learned more about physiotherapy through the referral process. The majority of respondents agreed that the appropriate career path for an animal physiotherapist was through qualification as a chartered physiotherapist. Postgraduate courses and Masters degrees in animal physiotherapy are available in the University of London and the University of Queensland.

Several respondents indicated that they had not referred as they did not know an animal physiotherapist in the area or they did not know how to find one. Greater communication and dissemination of information is essential if veterinary surgeons are to be informed of the role and availability of the animal physiotherapist. In this study, 7I (92\%) respondents called for the setting up of a register of animal physiotherapists, as exists in the UK. Active promotion of animal physiotherapy is also required within the physiotherapy profession. Clearly, animal physiotherapists also need to promote their own practices with veterinary colleagues in the locality. 
Currently, in Ireland and the UK, the law decrees that an animal physiotherapist may only treat an animal if it has been referred by a veterinary surgeon (Ryan and Finn, 2000). The Association of Physiotherapists in Animal Therapy (ACPAT, 1999) is a Clinical Interest Group of the Chartered Society of Physiotherapy and was founded in 1985 following concerns raised by UK veterinary surgeons regarding the number of unqualified animal physiotherapists (Bromiley, 1993), and has 178 members. Category A members are allowed to perform unsupervised physiotherapy on animals. Category B members are not necessarily physiotherapists and must be supervised by a category A member or a veterinary surgeon. There is no Irish equivalent of ACPAT.There are only five or six chartered physiotherapists practicing animal physiotherapy in Ireland (Ryan, 200I).

The majority of respondents $(n=74: 96 \%)$ called for more research on the effect of animal physiotherapy. If animal physiotherapy is to develop, ongoing research is essential to increase the evidence base for efficacy of treatment. The publication of research in veterinary and physiotherapy journals could enhance awareness of animal physiotherapy and create a forum for discussion benefiting veterinary medicine and animal physiotherapy.

Many respondents (55\%) felt that cost did not deter owners from seeking treatment. Fifty-two respondents described competitive animals as being particularly appropriate to receive physiotherapy. The reduction in recovery time and return to full fitness is vital to protect a valuable asset. This economic aspect has not been examined in any depth in previous surveys of veterinary surgeons. Interestingly, a majority of the veterinary surgeons $(n=73 ; 93 \%)$ supported the concept of veterinary surgeons and animal physiotherapists working out of the same practice. Veterinary surgeons and animal physiotherapists could explore the economic and practical aspects of this arrangement in the future. Close liaison between veterinary surgeons and physiotherapists may avoid unnecessary referrals and promote referrals where the costs of animal physiotherapy are clearly justified.

The authors acknowledge some limitations of the present study. The response rate was somewhat low with only $49 \%$ of questionnaires returned and the possibility of a non-response bias exists. Stratification of the sample for geographical region and type of veterinary practice did not occur and, therefore, it is not entirely evident how representative the 97 respondents are of those currently practising veterinary surgery in Ireland.

\section{Conclusion}

The majority of Irish veterinary surgeons recognised that the animal physiotherapist can have a role in modern veterinary medicine. However, for animal physiotherapy to be widely used, veterinary surgeons need to have knowledge of, and access to, chartered animal physiotherapists. Communication between the two professions is essential. Animal physiotherapy is in its infancy in Ireland. It is vital that animal physiotherapists demonstrate the effectiveness of treatments among veterinary colleagues if this specialist area of physiotherapy is to progress.

\section{Acknowledgements}

We wish to thank all of the veterinary surgeons who participated in the study and the Veterinary Council of Ireland for allowing us to use the Veterinary Register.

\section{References}

Association of Chartered Physiotherapists in Animal Therapy (ACPAT) (1999). Standards document.

Antikatzides, T. (1986). Soft laser treatment of musculoskeletal and other disorders in the equine athlete. Equine Practice 8: 24-30.

Bromiley, M. (1991). Physiotherapy in Veterinary Practice. London: Blackwell Scientific.

Bromiley, M. (1993). Equine Injury, Therapy and Rehabilitation. Oxford: Blackwell Scientific.

Bromiley, M. (1994). Physiotherapy for equine injuries. Equine Veterinary Education 6: 24I-244.

Cramp, F. (1998). The current use of ultrasound among category A equine physiotherapists in the United Kingdom. Journal of the Association of Chartered Physiotherapists in Animal Therapy 7: 26-27.

Herrod-Taylor, E. (1967). A technique for manipulation of the spine in horses. Veterinary Record 8I: 432.

Hodges, C.C. and Palmer, R.H. (1993). Post-operative physical therapy. In: Surgical Complications and Wound Healing in Small Animal Practice. Edited by J. Harari. Philadelphia: Saunders. Chapter 17, pp389-405.

Knowles, D. and Mackintosh, S. (1994). A survey of animal physiotherapy practice in Britain. Physiotherapy 80: 285-289.

Lang, C. (1980). Ultrasonic treatment of musculoskeletal conditions in the horse, dog and cat. Veterinary Record 106: 427-435.

McNamara, K. and Mackintosh, S. (1993). Veterinary surgeons' perceptions of animal physiotherapy. Physiotherapy 79: 312-316.

Mills, D. and Levine, D. (1997). The role of exercise and physical modalities in the treatment of osteoarthritis. Veterinary Clinics of North America: Small Animal Practice 27: 913-930.

Morcos, M. and Aswad, A. (1978). Treatment of two clinical conditions in racehorses by ultrasonic therapy. Equine Veterinary Journal I0: I28- 129.

Marr, C.M., Love, S., Boyd, J.S. and McKellar, Q. (1993). Factors affecting the clinical outcome of injuries to the superficial digital flexor tendon in national hunt and point-to-point racehorses. Veterinary Record 132: 476-479.

O'Connor, M. (1988). Physiotherapy in equine practice. Equine Sports Medicine News 7: 101-102.

Porter, M. (1988). What is physical therapy for horses? Equine Sports Medicine News 7: 24-28.

Ryan, T. (200I). Animal physiotherapy - a brief overview. Physiotherapy Ireland 22: 9-II.

Ryan, T. and Finn, A. (2000). Observations on a survey of veterinary students' perceptions of animal physiotherapy. Irish Veterinary Journal 53: |43-145.

Sharifi, D. and Sharma, S. (1990). Effect of physiotherapy after tibial transfixation in cattle. Indian Veterinary Journal 67: 952-954.

Shipley, G. (1999). A survey into veterinary surgeons' awareness, knowledge and use of animal physiotherapy. Journal of the Association of Chartered Physiotherapists in Animal Therapy 2: 47-48.

Taylor, G. (1970). Treating elephants with short-wave diathermy. Physiotherapy 56: 62-64.

White, A. and White, V. (1995). Physiotherapy as an aid to treatment of bilateral femoral paralysis in a calf. Veterinary Record I37: 489-491. 